\title{
Refractory High Grade B-Cell Lymphoma
}

National Cancer Institute

\section{Source}

National Cancer Institute. Refractory High Grade B-Cell Lymphoma. NCI Thesaurus.

Code C160233.

High grade B-cell lymphoma that is resistant to treatment. 